The Astrophysical Journal, 271:702-716, 1983 August 15

(C) 1983. The American Astronomical Society. All rights reserved. Printed in U.S.A.

\title{
STATIONARY FLOWS IN THE CIRCUMSTELLAR ENVELOPES OF M GIANTS
}

\author{
A. G. G. M. TIELENS \\ Sterrewacht Leiden, Leiden, The Netherlands \\ Received 1982 September 17; accepted 1983 February 4
}

\begin{abstract}
We have developed a model for a stellar wind driven by radiation pressure on dust grains in the circumstellar envelopes of Mira variables. This theory couples for the first time the gas temperature and the velocity variations. This coupling forms an essential aspect of the flow. The coupling is present because the escape of cooling photons depends on the local velocity gradient. The topology of the solution of the momentum equation of the gas is described, and it is shown that a critical point exists in the flow, related to the sonic point in the familiar solar wind theory. A solution which connects smoothly the region of subsonic flow close to the inner boundary with the region of supersonic flow at the outer boundary has to pass through this point. Finally, the flow for a typical Mira variable is calculated numerically, and its characteristics are briefly discussed.
\end{abstract}

Subject headings: stars: circumstellar shells - stars: late-type — stars: long-period variables stars: winds

\section{INTRODUCTION}

For 25 years we have known from optical studies that $\mathrm{M}$ giants are losing mass at a high rate $\left(>3 \times 10^{-8} M_{\odot}\right.$ $\mathrm{yr}^{-1}$; Deutsch 1956). However, the metallic absorption lines and hydrogen emission lines in the optical spectra show complex time variations which prevent a simple interpretation in terms of a constant expanding envelope (Wallerstein 1975).

During the last decade our knowledge of the circumstellar envelopes around these stars has increased considerably. First, broad-band infrared observations show emission in excess of the expected stellar continuum (Merrill 1977). This emission is attributed to dust grains condensing in the outflowing gas. In this article we will concentrate on oxygen-rich Mira variables. The infrared spectra of these stars show two broad emission features, one at $1030 \mathrm{~cm}^{-1}(9.7 \mu \mathrm{m})$ and one at $570 \mathrm{~cm}^{-1}(17.5 \mu \mathrm{m})$. These are generally believed to be due to the stretching and bending vibration in silicate material (Gehrz and Woolf 1971; Merrill 1977; Forrest et al. 1978). However, no infrared spectrum of terrestrial minerals, moon rocks, meteorites, or laboratory-made amorphous silicates has yet reproduced both features satisfactorily (Pollack, Toon, and Khare 1973; Penman 1976; Day 1979). Lunar occultation measurements and infrared interferometry show that the dust emission starts only at 5-10 stellar radii (Toombs et al. 1972; Sutton et al. 1978).

Second, maser emission of $\mathrm{OH}, \mathrm{H}_{2} \mathrm{O}$, and SiO molecules has been detected toward many of these stars. Line observations of the $\mathrm{OH}$ maser-which is the best studied-yield expansion velocities of circumstellar en- velopes between 5 and $20 \mathrm{~km} \mathrm{~s}^{-1}$ (Winnberg 1977). The $\mathrm{OH}$ line fluxes vary simultaneously with the infrared fluxes. However, small differences between the phases of the $\mathrm{OH}$ light curves from the front and from the back of the expanding envelope yield a typical distance between the star and the masing region of about $10^{16}-10^{17} \mathrm{~cm}$ (Jewell, Webber, and Snyder 1980; Herman and Habing 1981). The $\mathrm{H}_{2} \mathrm{O}$ and $\mathrm{SiO}$ masing region is much smaller, typically about $10^{14} \mathrm{~cm}$ (Spencer et al. 1979; Moran et al. 1979).

Third, recently absorption and thermal emission lines of $\mathrm{CO}, \mathrm{NH}_{3}, \mathrm{SiO}$, and $\mathrm{H}_{2} \mathrm{O}$ molecules have been detected at millimeter and infrared wavelengths (Morris et al. 1979; Lo and Bechis 1977; Zuckerman et al. 1976, 1978; Betz and McLaren 1980; Bernat et al. 1979; Hinkle 1978; Hinkle and Barnes 1979; Hinkle, Hall, and Ridgway 1982). In particular, the near-infrared absorption lines of $\mathrm{CO}$ and $\mathrm{H}_{2} \mathrm{O}$ molecules yield insight into the structure of the inner parts of the expanding envelope. The picture emanating from these data is that three different regions are to be recognized: (1) an extended pulsating photosphere terminated by (2) a stationary layer from which starts (3) the cool expanding envelope (Hinkle, Hall, and Ridgway 1982).

Oscillations of the star drive strong waves into the photosphere. Shock fronts form at the interface of outward driven material and material falling inward from previous cycles. As inferred from their velocity behavior, the hydrogen emission lines are formed in the shocked gas, while the metallic absorption lines are formed in the material falling back to the star. This observational picture is corroborated by extensive shock model calcu- 
lations of a pulsating photosphere (Wood 1979; Willson and Hill 1979; Hill and Willson 1979). In this way material is transported to large distances $\left(5-10 R_{\star}\right)$ from the star, where it forms a stationary layer.

This layer acts as a reservoir from which material drains back to the star and from which material is accelerated outward. The $\mathrm{SiO}$ maser probably forms at the outer layer of this stationary shell. Dust particles condense in the stationary layer. Radiation pressure by stellar photons accelerates the grains outward. Because of gas-grain collisions the gas is dragged along (Gilman 1972). In this way a cool and expanding envelope is formed that is detected in the $\mathrm{OH}$ maser lines and the millimeter and infrared molecular absorption and emission lines.

This rather qualitative picture requires further theoretical and observational confirmation. For example, there is no useful observational or theoretical tool to connect the observed velocity or temperature to a definite distance from the stellar surface. This absence of radial information prevents, for example, an accurate determination of mass loss rates. The existing estimates of mass loss rates are often uncertain by a factor of 10 or more. Since the mass loss rate influences the further evolution of the star, a more accurate determination appears quite important. Model calculations of the gas flow in the circumstellar envelopes of $M$ giants are thus important. However, they hardly exist.

Several theoretical studies have been devoted to the physics and chemistry of circumstellar shells around late-type stars. The formation of dust grains has been examined in some detail by Salpeter (1974a, $b$ ), Kwok (1975), Draine and Salpeter (1977), and Deguchi (1980). The infrared continuum has been modeled by Jones and Merrill (1976), Bedijn (1977), and Menietti and Fix (1978). Chemistry models have been made by Tsuij (1973), Goldreich and Scoville (1976), Scalo and Slavski (1980), and Clegg, van IJzendoorn, and Allamandola (1982). Maser models have been proposed by Elitzur, Goldreich, and Scoville (1976) for the $\mathrm{OH}$ maser and by Watson, Elitzur, and Bieniek (1980) for the SiO maser. The dynamics of the outflow have been studied by Kwok (1975), Goldreich and Scoville (1976), Olnon (1977), and Menietti and Fix (1978). Because of the complicated nature of the dynamical problem, simplifying assumptions had to be made. In particular, in all of the model calculations it has been assumed that the temperature and velocity of the gas do not influence each other and can thus be studied separately. This assumption defies an essential aspect of the dynamical problem. As will be shown in this article, the temperature and velocity of the gas are closely coupled. The cooling of the gas is dominated by the escape of photons produced in radiative decay of collisionally excited levels of abundant molecules, in particular $\mathrm{H}_{2} \mathrm{O}$ (Goldreich and Scoville 1976). The escape probability of the pho- tons depends on the local value of the velocity gradient. There is therefore a direct coupling between the temperature of the gas and the gradient of its velocity. Mathematically, the dependence of the cooling on the velocity gradient introduces a nonlinearity in the momentum equation of the gas that resembles one encountered in radiatively driven winds in Of stars (Castor, Abbott, and Klein 1975). It can be solved numerically in the same way. Similarly to flows from Of stars, there is a critical point in the flow of cool circumstellar envelopes. A solution which connects smoothly the region of subsonic flow at the inner boundary with the region of supersonic flow at the outer boundary has to pass through such a critical point.

This article is organized as follows. In § II we outline the theory of a stellar wind, driven by radiation pressure on grains. The new, and essential, feature of the dynamics - the dependence of the cooling on the local velocity gradient - is described in some detail. Properties of the resulting nonlinear differential equation and a method to solve this equation are delineated in $\S$ III. In $\S$ IV the results of the numerical integration for one particular case are presented and discussed. Finally, in $\S \mathrm{V}$ the main results of our work are summarized.

\section{THEORY OF A STELLAR WIND DRIVEN BY RADIATION PRESSURE ON GRAINS}

Dust particles condensate in the stationary layer. Radiation pressure on the dust particles accelerates them outward. The gas is dragged along by collisions with the dust. The mean free path of a gas atom between two collisions with a grain is large compared with the dimensions of the system. The dust and gas are therefore not positionally coupled (Weyman 1962). There are, however, enough collisions of gas atoms with a dust particle to transfer essentially all the momentum which the dust particle gains from the radiation field to the colliding gas atoms. Internal collisions of the gas redistribute this momentum over all gas particles. The dust and gas are therefore momentum coupled (Gilman 1972).

We are thus considering a two-component fluid, consisting of dust and gas particles. Each component has its own density, temperature, and velocity structure. The two components are coupled through the dust-gas collisions which transfer momentum and energy from the dust to the gas.

We want to calculate the stationary, that is, steady in time, solutions of the flow in the circumstellar envelopes of late-type stars. In particular, we are interested in those flows which start subsonically at the stationary layer and become smoothly, that is, without shocks, supersonic far from this layer. Furthermore, we will assume that the flow is radial and spherically symmetric. For each component we can write three equations de- 
scribing conservation of mass, momentum, and energy. We will first consider the dust component and then the gas component.

\section{a) The Dust Component}

We will assume that the grains condensate instantaneously in the stationary layer. All the grains are assumed to have the same radius, $a$, and the same velocity, $v_{\text {g }}$. The mean free path between grain-grain collisions is large compared with the dimensions of the system. There is therefore no internal interaction within the dust component and, consequently, no kinetic temperature. A dust grain has, of course, an excitation temperature which is largely determined by absorption and reemission of radiation. This temperature will not be considered further, as it has no influence on the question we want to answer. There are therefore two equations describing the flow of the dust component. The equation of continuity

$$
\dot{\Re}_{g}=4 \pi r^{2} n_{g} m_{g} v_{g}
$$

and the momentum equation per dust particle

$$
m_{g} v_{g} \frac{d v_{g}}{d r}=\frac{\sigma_{g} \bar{Q}_{\mathrm{rp}} L_{\star}}{4 \pi r^{2} c_{l}}-\frac{G M_{\star} m_{g}}{r^{2}}-F_{d},
$$

where $r$ is the radial coordinate; $\dot{\mathscr{T}}_{g}, n_{g}, m_{g}, v_{g}, \sigma_{g}$, and $\bar{Q}_{\text {rp }}$ are the mass loss rate, number density, mass, velocity, geometrical cross section, and flux-weighted mean of the radiation pressure efficiency of the grains; $L_{\star}$ and $M_{\star}$ are the stellar luminosity and mass; $G$ and $c_{l}$ are the gravitational constant and the velocity of light. The three terms on the right-hand side of equation (2) describe successively the radiation pressure force, the gravitational force, and the drag force $\left(F_{d}\right)$ between gas and dust. In the outflow of late-type giants the gravitational force on dust grains can be neglected in comparison to the radiation pressure force (Gilman 1972). It has been shown for the case of $\alpha$ Ori that the grains reach their local terminal velocity within a distance of $\sim 10^{11}$ $\mathrm{cm}$, which is small compared with the characteristic dimensions of the envelope, $10^{14}-10^{17} \mathrm{~cm}$ (Gilman 1972). We will therefore assume that negligible force is needed to accelerate the grains to their local terminal velocity. As discussed before, all the momentum which the grains gain from the radiation field is transferred to the gas through the gas-grain collisions. The detailed question of how the grains form and how they acquire their speed is, however, important for dense flows (large mass loss rates). We will come back to this point in § IV.

We can calculate the drift velocity, $v_{d}$, of the grains with respect to the gas in the following way $\left(v_{d}=v_{g}-v\right.$, where $v$ is the gas velocity). Following Kwok (1975), we will approximate the drag force on a grain by the following expression:

$$
F_{d}=\sigma_{g} \rho v_{d}\left(c^{2}+v_{d}^{2}\right)^{1 / 2},
$$

where $\rho$ and $c$ are the density and local sound velocity of the gas, respectively. Equation (3) has the correct limits for a drift velocity much larger and much smaller than the gas velocity. Because the radiation pressure on the grains equals the drag force, we then find for the drift velocity

$$
\left.v_{d}=\llbracket \frac{1}{2}\left\{\left[\left(\frac{\bar{Q}_{\mathrm{rp}} L_{\star}}{2 \pi c_{l} \rho r^{2}}\right)^{2}+c^{4}\right]^{1 / 2}-c^{2}\right\}\right]^{1 / 2} .
$$

Once we know the sound velocity (i.e., the temperature) and density of the gas, we can calculate the velocity and density of the dust from equations (1) and (4).

\section{b) The Gas Component}

The equations expressing conservation of mass, momentum, and energy of the gas are

$$
\begin{gathered}
\dot{\mathfrak{T}}_{\star}=4 \pi r^{2} \rho v, \\
v \frac{d v}{d r}+\frac{1}{\rho} \frac{d P}{d r}+\frac{G M_{\star}}{r^{2}}(1-\Gamma)=0,
\end{gathered}
$$

and

$$
\begin{gathered}
\frac{1}{r^{2}} \frac{d}{d r}\left\{\rho v r^{2}\left[\frac{1}{2} v^{2}+\left(\frac{\gamma}{\gamma-1}\right) \frac{P}{\rho}\right]\right\} \\
=-\rho v \frac{G M_{\star}}{r^{2}}(1-\Gamma)+q,
\end{gathered}
$$

where $\dot{N}_{\star}, v, P$, and $\gamma$ are the mass loss rate, velocity, pressure, and ratio of specific heats of the gas; $\Gamma$ is the ratio of the drag force to the gravitational force, to be discussed in $\S$ II $c(\mathrm{i}) ; q$ is the net heat added to the flow per unit volume.

Following Bondi (1952) and Holzer and Axford (1970), we introduce the following variables: the radial distance

$$
\xi \equiv r / r_{0},
$$

the Mach number

$$
M \equiv \frac{v}{c},
$$

the enthalpy

$$
H \equiv \frac{1}{2} v^{2}+\left(\frac{\gamma}{\gamma-1}\right) \frac{P}{\rho}=\frac{1}{2}\left(M^{2}+\frac{2}{\gamma-1}\right) c^{2},
$$


and the heat function

$$
Q \equiv 4 \pi r_{0}^{3} q(\xi) \xi^{2} / \dot{\Upsilon}_{\star} .
$$

Here $r_{0}$ is a free-to-choose reference radius, and we have used

$$
c^{2}=\gamma P / \rho .
$$

With these definitions, assuming $\gamma=5 / 3$ and using the ideal gas law

$$
P=\rho k T / \mu m_{\mathrm{H}}
$$

equations (6) and (7) can be rewritten into

$$
\begin{aligned}
\frac{M^{2}-1}{2 M^{2}} \frac{d M^{2}}{d \xi}=\frac{M^{2}+3}{3 H}[ & \frac{2 H}{\xi}-\frac{2 G M_{\star}}{r_{0} \xi^{2}}(1-\Gamma) \\
& \left.-\frac{5}{6}\left(M^{2}+\frac{3}{5}\right) Q\right]
\end{aligned}
$$

and

$$
\frac{d H}{d \xi}=-\frac{G M_{\star}}{r_{0} \xi^{2}}(1-\Gamma)+Q .
$$

It follows that the temperature gradient is given by

$$
\frac{d T}{d \xi}=\frac{2 \mu m_{\mathrm{H}}}{3 k} Q-\frac{4}{3} \frac{T}{\xi}\left(1+\frac{r}{2 v} \frac{d v}{d r}\right),
$$

and the heat function by

$$
Q=Q_{1}-Q_{2},
$$

where $Q_{1}$ is the viscous heating of the gas due to gas-grain collisions, and $Q_{2}$ is the cooling through escape of photons.

\section{c) Gas-Grain Coupling}

The gas and grains are coupled by the momentum and energy transfer in gas-grain collisions. In the flow equations of the gas this coupling enters through the ratio of the drag force to the gravitational attraction, $\Gamma$, and through the viscous heating of the flow, $Q_{1}$. We will now first derive an expression relating $\Gamma$ to the flow variables, $\xi, M$, and $H$. Then we will do the same for $Q_{1}$.

\section{i) The Momentum Transfer}

All the momentum gained by the grains from the radiation field is transferred to the gas. Using equations (2) and (3), we find for $\Gamma$ the following expression:

$$
\Gamma=\frac{\sigma_{g} \bar{Q}_{\mathrm{rp}} L_{\star}}{4 \pi c_{l} G M_{\star}} \frac{n_{g}}{\rho} .
$$

Using the continuity equations for the dust (eq. [1]) and for the gas (eq. [5]), we may write

$$
\Gamma=\frac{\sigma_{g} \bar{Q}_{\mathrm{rp}} L_{\star} \delta}{4 \pi c_{l} G M_{\star} m_{g}} \frac{M}{M_{g}},
$$

where $\delta$ is the dust-to-gas mass loss ratio, and $M_{g}$ is defined by

$$
M_{g} \equiv \frac{v_{g}}{c}=\frac{v+v_{d}}{c} \equiv M+M_{d},
$$

and $M_{d}$ is given by (cf. eq. [4])

$$
M_{d}=\llbracket \frac{1}{2}\left\{\left[\gamma_{d} M^{2} \frac{\left(M^{2}+3\right)}{2 H}+1\right]^{1 / 2}-1\right\} \rrbracket^{1 / 2},
$$

where

$$
\gamma_{d}=\left(\frac{2 \bar{Q}_{\mathrm{rp}} L_{\star}}{\dot{\mathfrak{T}}_{\star} c_{l}}\right)^{2}
$$

With equations (11) and (12), $\Gamma$ is related to the variables $M$ and $H$.

\section{ii) Energy Transfer from Dust to Gas}

The most important heat source of the gas is viscous heating (Goldreich and Scoville 1976). We will approximate the heat added to the flow by this process by

$$
q_{1}=\frac{1}{2} \rho n_{g} \sigma_{g} v_{d}^{3} .
$$

For the heat function $Q_{1}$ we then find

$$
Q_{1}=\left(\frac{\dot{\mathfrak{T}}_{\star}}{8 \pi r_{0}} \sigma_{g} \frac{\delta}{m_{g}}\right) \frac{M_{d}^{3}}{M M_{g} \xi^{2}}\left(\frac{2 H}{M^{2}+3}\right)^{1 / 2} .
$$

\section{d) Cooling by $\mathrm{H}_{2} \mathrm{O}$ Molecules}

$\mathrm{H}_{2} \mathrm{O}$ molecules are rotationally excited by collisions with $\mathrm{H}_{2}$ molecules. Subsequent radiative decay will contribute to the cooling of the gas when the photon escapes from the circumstellar envelope. Although other molecules undoubtedly contribute to the cooling, the $\mathrm{H}_{2} \mathrm{O}$ cooling is the most important (Goldreich and Scoville 1976). We introduce the cooling equations in a two-step process. First, we assume that the $\mathrm{H}_{2} \mathrm{O}$ molecules have an excitation temperature, $T_{x}$, which is less than the kinetic temperature of the gas. This leads to an expression for the cooling rate. Second, we relate the ratio $T_{x} / T$ to the escape probability of the photons.

\section{i) The Cooling Rate}

Calculating the populations of the many $\mathrm{H}_{2} \mathrm{O}$ rotational levels is beyond the scope of this model. Instead 
we will follow Goldreich and Scoville (1976) in representing $\mathrm{H}_{2} \mathrm{O}$ by a classical model molecule which contains, however, the essential features of the $\mathrm{H}_{2} \mathrm{O}$ rotational cooling. We assume that the model molecule has two excited rotational levels in the ground vibrational state. These levels are separated by an energy difference $h \nu$ and are both situated at an energy of about $3 k T_{x} / 2$ above the ground rotational state. Where $T_{x}$ is the excitation temperature of the model molecules, generally $h \nu \ll 3 k T_{x} / 2$. The heat loss rate per unit volume is given by the difference between excitation and de-excitation collisions between the two levels.

$$
\begin{aligned}
q_{2}=n_{\mathrm{H}_{2}} n_{\mathrm{H}_{2} \mathrm{O}}\langle\sigma v\rangle h \nu[ & \exp (-h \nu / k T) \\
- & \left.\exp \left(-h \nu / k T_{x}\right)\right] .
\end{aligned}
$$

Here $n_{\mathrm{H}_{2}}$ and $n_{\mathrm{H}_{2} \mathrm{O}}$ are the number density of $\mathrm{H}_{2}$ and $\mathrm{H}_{2} \mathrm{O}$. For the collision rate constant, $\langle\sigma v\rangle$, we assume that

$$
\langle\sigma v\rangle=c_{0} T^{1 / 2},
$$

with $c_{0}$ a constant. For the frequency, $\nu$, we take the classical value of the rotational frequency of an $\mathrm{H}_{2} \mathrm{O}$ molecule with a rotational energy of $3 k T_{x} / 2$ :

$$
\nu=\nu_{0} T_{x}^{1 / 2},
$$

with $\nu_{0}$ a constant (Goldreich and Scoville 1976; see Appendix). Assuming $h \nu \ll k T$ and $h \nu \ll k T_{x}$ and introducing

$$
x \equiv T / T_{x},
$$

we may write for the heat loss due to this process

$$
\begin{aligned}
Q_{2}= & \frac{\dot{M}_{\star}}{8 \pi r_{0}} c_{0}\left(A_{\mathrm{O}}-A_{\mathrm{C}}\right)\left(h \nu_{0}\right)^{2}\left(\frac{3 \mu}{k^{3} m_{\mathrm{H}}^{3}}\right)^{1 / 2} \\
& \times\left(\frac{M^{2}+3}{2 H}\right)^{1 / 2} \frac{1-(1 / x)}{M^{2} \xi^{2}},
\end{aligned}
$$

where $\mu$ is the mean molecular weight, and $A_{\mathrm{O}}$ and $A_{\mathrm{C}}$ are the elemental abundances of oxygen and carbon, respectively. We have assumed that all of the oxygen which is not in the form of $\mathrm{CO}$ is in the form of $\mathrm{H}_{2} \mathrm{O}$. Note that cooling occurs when $x>1$, i.e., when $T>T_{x}$.

Equation (16) relates the $\mathrm{H}_{2} \mathrm{O}$ rotational cooling of the gas to the variables $\xi, H$, and $M$ and to the excitation temperature, $T_{x}$.

\section{ii) The Excitation Temperature of the Rotational Levels of $\mathrm{H}_{2} \mathrm{O}$}

We will now relate the excitation temperature, $T_{x}$, to the flow variables. The two rotational levels are connected by collisional excitation and de-excitation and radiative decay. For simplicity we have neglected radiative excitation induced by stellar photons. For the same reason we have neglected radiative excitation to higher vibrational levels followed by radiative de-excitation to excited rotational levels in the ground vibrational state. At the low temperatures of the flow $(T<1000 \mathrm{~K})$ collisional excitational to higher vibrational levels can be ignored.

Only those rotational photons that escape from the local surroundings of the emitting molecule count for the net cooling of the gas, that is, for making $T_{x}<T$. In order to calculate the net radiative decay we have therefore to solve the radiative transfer equation in the line, in principle, an enormous task. However, in an expanding envelope the velocity gradient dominates the photon escape and thermalization process (Sobolev 1960). The source function is then geometrically localized, and the solution of the radiative transfer equation is replaced by the calculation of escape probabilities. Defining $\beta_{21} A_{21}$ as the net radiative decay rate of the higher level (2), we can write for the rate equation determining the level populations in equilibrium

$$
\frac{d n_{1}}{d t}=\beta_{21} A_{21} n_{2}-\langle\sigma v\rangle\left[n_{1} \exp \left(\frac{-h \nu}{k T}\right)-n_{2}\right]=0,
$$

and

$$
n=n_{1}+n_{2} \text {, }
$$

where the $n_{i}$ 's are the population of level $i$, and $n$ is the total molecular number density. The $\mathrm{H}_{2} \mathrm{O}$ rotational transitions are optically thick (Goldreich and Scoville 1976). The escape probability is then given by (Castor 1970)

$$
\beta_{21}=\frac{8 \pi v}{3 h c_{l} r B_{12}\left(n_{1}-n_{2}\right)}\left(1+\frac{1}{2} \varepsilon\right),
$$

where $B_{12}$ is the Einstein transition probability, and $\varepsilon$ is given by

$$
\varepsilon=\frac{r}{v} \frac{d v}{d r}
$$

Using some approximations discussed in the Appendix we can rewrite equations (16) and (17) into

$$
g_{x} M^{3} \xi^{3}\left(\frac{2 H}{M^{2}+3}\right)\left(1+\frac{1}{2} \varepsilon\right)=x^{5 / 2}(x-1),
$$

where $g_{x}$ is a constant. Equation (21) demonstrates that the excitation temperature (and thus the cooling law) depends on the local velocity gradient, $\varepsilon$, and, of course, on the variables $\xi, H$, and $M$. This is the essence of the coupling between the $\mathrm{H}_{2} \mathrm{O}$ rotational cooling and the gas flow. 


\section{PROPERTIES AND METHOD OF SOLUTION OF THE MOMENTUM EQUATION OF THE GAS}

In order to find the velocity and density structure of the gas and dust and the temperature structure of the gas, we have to solve the two differential equations (8) and (9) (i.e., the momentum and energy equation of the gas) together with the three algebraic equations (1), (12), and (5) (i.e., the continuum and momentum equation of the grains and the continuum equation of the gas). Because the $\mathrm{H}_{2} \mathrm{O}$ rotational cooling depends on the velocity gradient (eq. [21]), the momentum equation for the gas (eq. [8]) is nonlinear, and the familiar theory of the solar wind (Parker 1960; Holzer and Axford 1970) is no longer applicable. It may be profitable to think of the solution to the flow equations as a one-dimensional curve in the three-dimensional space of $\left(\xi, H, M^{2}\right)$; these three dimensions represent, respectively, $(r, T, v)$. We require that along this curve $\varepsilon(\equiv d \ln v / d \ln \mathrm{r})$ is continuous. Equation (10) shows that $d T / d r$ is then automatically continuous. The momentum equation can be recast in the following schematic form

$$
\begin{aligned}
G= & \frac{H\left(M^{2}-1\right)}{\xi} \varepsilon-F\left(\xi, H, M^{2}\right) \\
& -h_{1}\left(\xi, H, M^{2}\right)\left(1-\frac{1}{x}\right)=0,
\end{aligned}
$$

where we have used (see eq. [20])

$$
\varepsilon=\frac{\xi}{2 H} \frac{d H}{d \xi}+\frac{3 \xi}{2 M^{2}\left(M^{2}+3\right)} \frac{d M^{2}}{d \xi}
$$

and $F$ and $h_{1}$ are defined by

$$
F \equiv \frac{2 H}{\xi}-\left(\frac{M^{2}+3}{2}\right) \frac{G M_{\star}}{r_{0}}\left(\frac{1-\Gamma}{\xi^{2}}\right)-\left(\frac{M^{2}+3}{3}\right) Q_{1}
$$

and

$$
h_{1} \equiv \frac{g_{2}}{3} \frac{\left(M^{2}+3\right)^{3 / 2}}{H^{1 / 2} M^{2} \xi^{2}}
$$

where $g_{2}$ is a constant. The equation, $G=0$, is a differential equation in $\varepsilon=d \ln v / d \ln r$. It is nonlinear because of the dependence of $x$ on $\varepsilon$ (see eq. [21]). The momentum equation written in the form of equation (22) is quite similar to the momentum equation encountered in the theory of radiatively driven winds in Of stars (Castor, Abbott, and Klein 1975), despite the vast difference in physics of the two problems. We will follow their discussion for our equation to arrive at the correct way to integrate the differential equation. a) The Existence of a Critical Point in the Flow

There exists a critical point in the flow through which a stationary solution should pass. If one solves the differential equation (22), starting either at the stationary layer, the sonic point, or at large distances, one encounters numerical problems. These numerical problems can be understood by analyzing the structure of equation (22) in a way similar to Castor, Abbott, and Klein (1975; see also Cassinelli 1979). Introducing the functions $G_{1}$ and $G_{2}$,

$$
G_{1} \equiv \frac{H\left(M^{2}-1\right)}{\xi} \varepsilon-F\left(\xi, H, M^{2}\right),
$$

which is linear in $\varepsilon$, and

$$
G_{2} \equiv h_{1}\left(\xi, H, M^{2}\right)\left(1-\frac{1}{x}\right)
$$

which depends in a nonlinear way on $\varepsilon$ through $x$, the differential equation, $G=0$, can be written as

$$
G=G_{1}-G_{2}=0
$$

Considerable insight into the behavior of equation (22) can be gained by considering a diagram of $G_{1}$ and $G_{2}$ as functions of $\varepsilon$ for a given set of $\xi, H$, and $M^{2}$ (Abbott 1977; Cassinelli 1979). The function $G_{1}(\varepsilon)$ is a straight line. The slope and intercept depend on the value of the variables $\xi, H$, and $M^{2}$. The function $G_{2}(\varepsilon)$ is a concave function of $\varepsilon$ which is zero for $\varepsilon=-2$, and which approaches asymptotically the value $h_{1}$ for large $\varepsilon$. The functions $G_{1}$ and $G_{2}$ change continuously in moving outward, that is, for increasing $\xi$, but $G_{2}$ always has a form similar to that sketched in Figure 1. In the solution of the differential equation $G=0$, five cases (a through e) can be distinguished depending on the slope and intercept of $G_{1}$ relative to $G_{2}$. These different forms of $G_{1}$ relative to $G_{2}$ are sketched in Figures $1 a, 1 b$, and $1 c$. The solution(s) of the differential equation (22) is given by the intersection(s) of $G_{1}$ with $G_{2}$. The five cases are identified in Table 1. Obviously, equation (22) can have none ( $d$ and e), one (a and c), or two (b) solutions. Close to the stationary layer the outflow will be subsonic $\left(M^{2}<1\right)$, and the solution is similar to case a (Fig. $1 a$ and Table 1). Far from the stationary layer the flow is supersonic $\left(M^{2}>1\right)$, and the intercept of $G_{1}(-F)$ becomes negative. The solution of equation (22) is then similar to case c (Fig. 1c, Table 1). For a continuous transition from case a to case $c$ the solution has to pass through case $\mathrm{b}$ (Fig. $1 b$, Table 1). The leftward root of case $b$ is continuously connected to the solution in case a while the rightward root is continuously connected to case c. Consequently, for a continuous transition from case a to case c, the straight line $G_{1}$ should at some point $\left(\xi, H, M^{2}\right)$ become tangential to the curve $G_{2}$. 


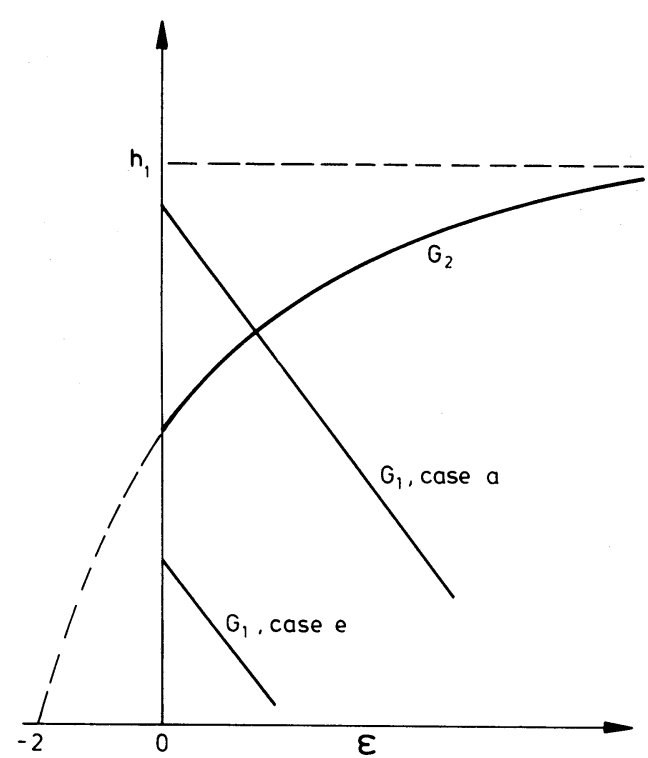

FIG. $1 a$

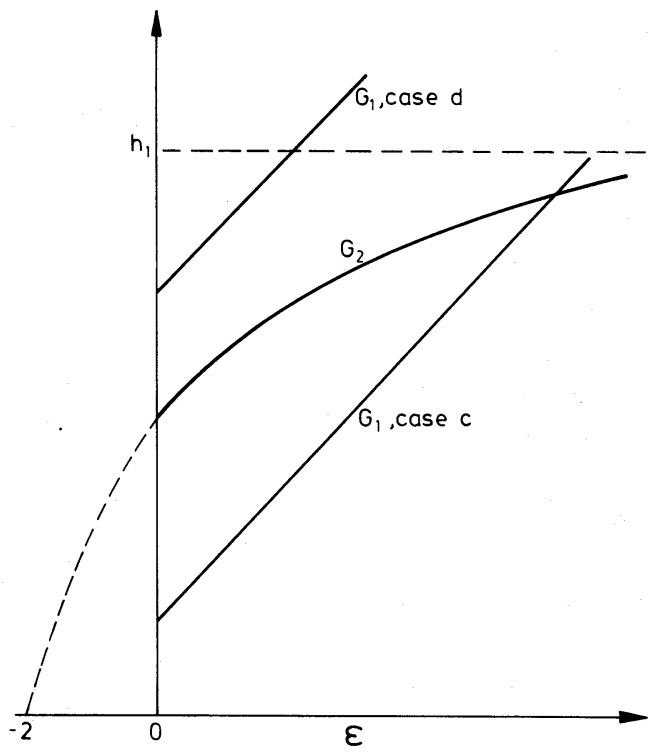

FIG. $1 c$

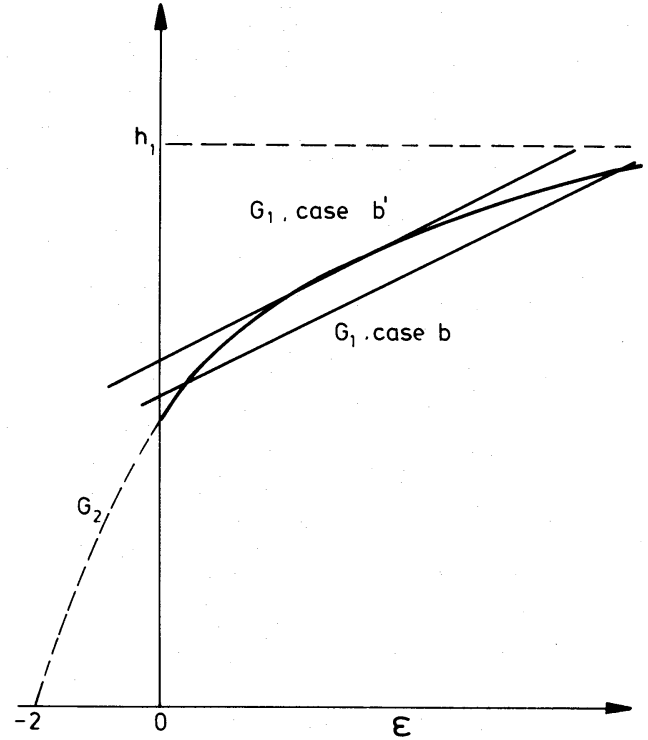

FIG. $1 b$

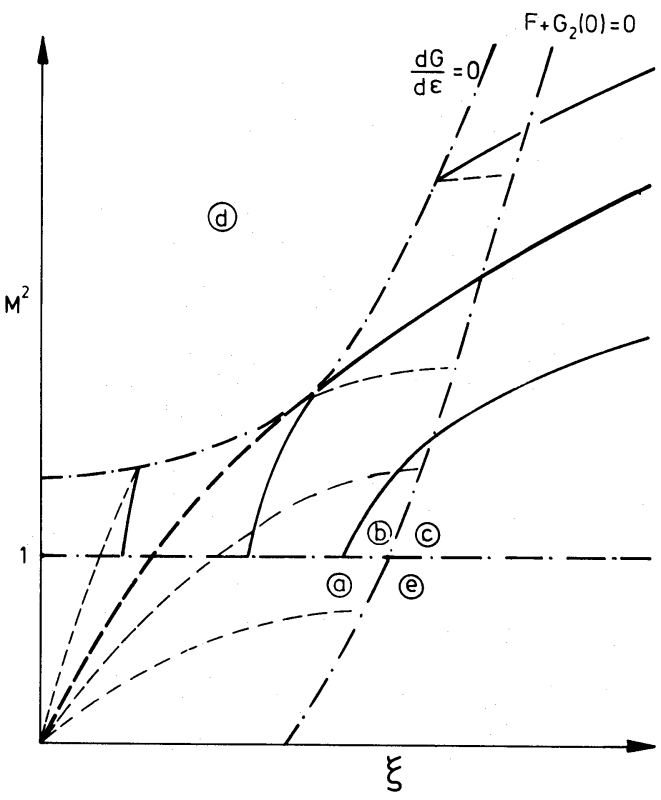

FIG. $1 d$

FIG. 1.- $(a-c)$ Graphical solution of the momentum equation of the gas (eq. [23]). The curved line shows $G_{2}$. The straight lines indicate several forms of $G_{1}$ (a through e). The intersection of $G_{1}$ with $G_{2}$ is the solution of the momentum equation of the gas. $(d)$ Topology of the solutions of the momentum equation of the gas in the $\left(\xi, M^{2}\right)$-plane. For simplicity the dependence on $H$ is ignored. The dashed-dotted lines divide the plane into five distinct regions (a through e) corresponding to the different graphical solutions displayed in Figs. la-l $c$. The dashed lines are leftward solutions of case $b$ which connect smoothly to case $a$. The solid lines are rightward solutions of case $b$ which connect smoothly to case $c$. The only solution which goes smoothly from subsonic velocities close to the inner boundary to supersonic velocities at the outer boundary is indicated by the heavy line. 
TABLE 1

Properties OF THE MOMENTUM EQUATION OF THE GAS

\begin{tabular}{ccc}
\hline \hline Case & \multicolumn{1}{c}{ Constraints } & $\begin{array}{c}\text { Number of } \\
\text { Solutions }\end{array}$ \\
\hline $\mathrm{a} \ldots \ldots$. & $M^{2} \leq 1 ; G_{2}(\varepsilon=0) \leq-F$ & 1 \\
$\mathrm{~b} \ldots \ldots$. & $M^{2}>1 ; G_{2}(\varepsilon=0) \leq-F$ & 2 \\
$\mathrm{~b}, \ldots \ldots$. & $M^{2}>1 ; G_{2}(\varepsilon=0) \leq-F ; d G / d \varepsilon=0$ & 2 \\
$\mathrm{c} \ldots \ldots \ldots$ & $M^{2}>1 ; G_{2}(\varepsilon=0)>-F$ & 1 \\
$\mathrm{~d} \ldots \ldots \ldots$ & $M^{2}>1 ; G_{2}(\varepsilon=0)<-F ; G>0$ & none \\
$\mathrm{e} \ldots \ldots \ldots$ & $M^{2} \leq 1 ; G_{2}(\varepsilon=0)>-F ; G<0$ & none \\
\hline
\end{tabular}

This condition is given by

$$
\frac{d G}{d \varepsilon} \equiv \frac{d G_{1}}{d \varepsilon}-\frac{d G_{2}}{d x} \frac{d x}{d \varepsilon}=0,
$$

where the derivatives are taken for constant, $\xi, H$, and $M^{2}$, and $d x / d \varepsilon$ follows from equation (21). The three equations (21), (22), and (24) define a singular locus, which is a two-dimensional topology because there are five unknowns $\left(\xi, H, M^{2}, \varepsilon\right.$, and $\left.x\right)$.

In Figure $1 d$ (adapted from Cassinelli 1979) we show the situation regarding the solution and possible abortive solution curves in the $\left(\xi, M^{2}\right)$-plane. This diagram gives a rather simplified impression, because the third dimension $(H)$ is left out. In this diagram a correct solution will be a continuous curve connecting the lower left corner with the upper right corner. The five cases mentioned before can be characterized by definite regions in the diagram. Regions $\mathrm{b}$ and $\mathrm{d}$ are separated by the singular locus. Regions $\mathrm{a}$ and $\mathrm{b}$ and regions $\mathrm{c}$ and $\mathrm{e}$ are separated by the plane $M^{2}=1$. Regions a and e and regions b and c are separated by the condition $G_{2}(\varepsilon=0)$ $+F=0$. These boundaries are indicated by the dashed-dotted lines in Figure $1 d$. For each $\xi, H$, and $M^{2}$ there exists one solution in region a which connects smoothly to the leftward root in region b. A few of these solution curves are indicated by dashed lines in Figure $1 d$. In region $\mathrm{c}$ there is also one solution which connects smoothly to the rightward root in region b. A few of these solution curves are indicated by solid lines in Figure $1 d$. In regions $\mathrm{d}$ and $\mathrm{e}$ there is no solution to the equation $G=0$. Obviously, there is only one point on the singular locus, hereafter called the critical point, which connects the solutions in regions a and c smoothly. In effect this is because the solution curve should become tangential to the singular locus in the critical point. Other solutions for the flow have a singular point at which the solutions terminate or show nodes or cusps. To find the condition for a continuous $\varepsilon$ we use

$$
\begin{aligned}
\frac{d G}{d \xi}= & \frac{\partial G}{\partial \xi}+\frac{\partial G}{\partial H} \frac{d H}{d \xi}+\frac{\partial G}{\partial M^{2}} \frac{d M^{2}}{d \xi} \\
& +\frac{\partial G}{\partial x} \frac{d x}{d \xi}+\frac{\partial G}{\partial \varepsilon} \frac{d \varepsilon}{d \xi}=0
\end{aligned}
$$

Using equation (24) and the condition that $d \varepsilon / d \xi$ should exist everywhere we find

$$
\begin{aligned}
\frac{\partial G}{\partial \xi}+ & \frac{\partial G}{\partial x} \frac{\partial x}{\partial \xi}+\left(\frac{\partial G}{\partial H}+\frac{\partial G}{\partial x} \frac{\partial x}{\partial H}\right) \frac{d H}{d \xi} \\
& +\left(\frac{\partial G}{\partial M^{2}}+\frac{\partial G}{\partial x} \frac{\partial x}{\partial M^{2}}\right) \frac{d M^{2}}{d \xi}=0
\end{aligned}
$$

The differentials $\partial x / \partial \xi, \partial x / \partial H$, and $\partial x / \partial M^{2}$ are found from equation (21). The derivatives $d M^{2} / d \xi$ and $d H / d \xi$ can be eliminated from equation (26) using equations (8) and (9). We are thus left with four equations $(21,22,24$, and 26$)$ in the five unknowns $(\xi, H$, $M^{2}, \varepsilon$, and $\left.x\right)$. For a stationary solution of the flow these four equations should be fulfilled in the critical point. Since there are more parameters than equations, there is still some freedom left. This implies that, apart from $\dot{M}_{\star}$, we are free to choose $\xi_{c}$.

The existence of a critical point is no surprise. In a flow without cooling, the flow must also fulfill special conditions at one special point. This is the so-called sonic point, where $M^{2}$ becomes equal to one. Obviously, for a smooth transition from subsonic to supersonic velocities the flow without cooling should fulfill the condition

$$
F\left(\xi, H, M^{2}\right)=0
$$

at the sonic point (cf. eqs. [8] or [22]). The acceleration at the sonic point is then found from equation (8) or equation (22) using l'Hospital's rule.

These conditions stem from the fact that the flow velocity becomes equal to the velocity of sound at the sonic point. In effect this point is the farthest point downstream which can still communicate with the flow upstream by exchanging pressure disturbances. Including cooling in the way described above makes it possible for the flow to communicate also by exchanging energy through the radiation field. The local velocity of communication lies therefore somewhere between the local velocity of sound and the velocity of light. This shifts the special point effectively from the sonic point to the critical point, farther downstream.

In the limit of vanishing cooling, equation (22) becomes the sonic condition, equation (24) is always fulfilled, and equation (26) becomes l'Hospital's rule applied to the momentum equation at $M^{2}=1$. Obviously, the rate equation, equation (21), does no longer apply. The familiar sonic condition for a flow without cooling is therefore a special case of the more general case described above.

\section{b) Solving the Flow Equations}

A calculation of the flow is started at the critical point. First, the conditions at the critical point are 
determined by solving equations (21), (22), (24), and (26) simultaneously. Then the differential equations (9) and (22) are integrated inward and outward. Special care is taken to ensure that the correct root of equation (22) is taken in region b. For a specific solution two boundary conditions have to be specified. In our calculations we have chosen to specify the stellar mass loss rate, $\dot{M}_{\star}$, and the radius of the critical point, $\xi_{c}$. The Mach number, enthalpy, velocity gradient, and excitation parameter at the critical point are then calculated from the conditions at the critical point (eqs. [21], [22], [24], [26]). In contrast, for a flow without cooling three boundary conditions need to be specified at the sonic point. This difference is due to the extra condition in our problem (eq. [24]). It stems from the nonlinearity of the differential equation, expressing the coupling between $T_{x}$ and $\varepsilon$. Once the conditions at the critical point have been determined, the differential equations (9) and (22) can be integrated inward to give the conditions at the inner boundary, in particular, the radius and temperature of the stationary layer. In reality these conditions are the natural boundary conditions, determined by the mass loss process in the stellar photosphere. The radius of the critical point and the mass loss rate will adjust themselves until the flow fulfills these boundary conditions. Thus, like the theory of radiatively driven winds in Of stars, the mass loss rate is an eigenvalue determined by the boundary conditions (Castor, Abbott, and Klein 1975). A similar situation exists in the theory of the solar wind, albeit that the constraint on the mass flux is less stringent than in our case (Couturier, Mangeney, and Souffrin 1979).

\section{RESULTS AND DISCUSSION}

In this section we will show that the method of solution described in $\S$ III is indeed capable of solving the problem. For this consistency check we will specify a set of parameters which enter into the calculations. We think that the chosen set is representative for oxygen-rich Mira variables. In a future publication we will investigate how variations in these parameters influence the flow and the observations thereof. The most important parameters are the parameters describing the properties of the dust. There are four of these: the flux-weighted mean of the radiation pressure efficiency, $\bar{Q}_{\mathrm{rp}}$, the size of the dust grain, $a$, the dust-to-gas mass ratio, $\delta$, and the specific weight of the dust particle, $\rho_{s}$. We have adopted the following values: $\bar{Q}_{\mathrm{rp}}=2 \times 10^{-2}, a=5 \times$ $10^{-6} \mathrm{~cm}, \delta=4 \times 10^{-3}$, and $\rho_{s}=2.5 \mathrm{~g} \mathrm{~cm}^{-3}$. The stellar mass and luminosity are fixed at $1 M_{\odot}$ and $10^{4} L_{\odot}$, respectively. The effective temperature of the star only enters through the flux-weighted mean of the radiation pressure efficiency and is implicit in our assumption of $\bar{Q}_{\text {rp }}$. The following parameters enter into the $\mathrm{H}_{2} \mathrm{O}$ rotational cooling of the gas: the moments of inertia of the
$\mathrm{H}_{2} \mathrm{O}$ molecule, $I_{1}, I_{2}$, and $I_{3}$, which are equal to $6.4 \times$ $10^{-40}, 1.2 \times 10^{-39}$, and $1.76 \times 10^{-39} \mathrm{~g} \mathrm{~cm}^{2}$, respectively (Townes and Schawlow 1955); and the collision cross section $\langle\sigma v\rangle$, which is set equal to $2 \times 10^{-11} T^{1 / 2}$ $\mathrm{cm}^{3} \mathrm{~s}^{-1}$ (Goldreich and Scoville 1976). For the abundance of carbon and oxygen we have adopted the cosmic abundance values. For simplicity in the discussion $r_{0}$ is set equal to one.

For the two boundary conditions, the mass loss rate, $\dot{M}_{\star}$, and the radius of the critical point, $\xi_{c}$, we have chosen the values $1.4 \times 10^{19} \mathrm{~g} \mathrm{~s}^{-1}\left(\sim 2 \times 10^{-7} M_{\odot} \mathrm{yr}^{-1}\right)$ and $1.7 \times 10^{14} \mathrm{~cm}\left(\sim 3.5 R_{\star}\right.$ for a star with an effective temperature of $2500 \mathrm{~K}$ ). The conditions at the critical point, which are determined from equations (21), (22), (24), and (26), are given in Table 2. In Figures 2, 3, and 4 we give the velocities $\left(v, v_{d}\right.$, and $\left.M^{2}\right)$, the densities $\rho$ and $\rho_{d} / \rho$, and the temperature $T$ as a function of $\xi$ for this model. The conditions at the inner boundary, that is, where the velocity becomes $5 \%$ of the sound velocity, are given in Table 3.

\section{a) Velocity Structure}

The velocities increase rapidly from the stationary layer outward. At about 10 times the inner radius the terminal velocities have already been reached (see Fig. 2). This is in agreement with earlier work (Kwok 1975). The Mach number continues to rise beyond this point because of the decrease in temperature and will ultimately go to infinity. The calculated value of the terminal velocity of the gas $\left(12 \mathrm{~km} \mathrm{~s}^{-1}\right)$ lies in the range $5-20 \mathrm{~km} \mathrm{~s}^{-1}$, typically observed for $\mathrm{OH}$ masers associated with oxygen-rich Mira variables (Dickinson and Chaisson 1973; Dickinson, Kollberg, and Yngnesson 1975; Olnon 1977). The terminal velocity depends strongly on the value chosen for $\xi_{c}$. The velocity of the grains is much larger than the gas velocity. The large drift velocity is due to the low density of the circumstellar envelope and the high radiation pressure efficiency of the dust.

TABLE 2

Conditions at the Critical Point

\begin{tabular}{ll}
\hline \hline$\xi_{c} \ldots \ldots \ldots \ldots$ & $1.74 \times 10^{14}$ \\
$M_{c}^{2} \ldots \ldots \ldots \ldots$ & 2.32 \\
$H_{c} \ldots \ldots \ldots \ldots$ & $1.71 \times 10^{11} \mathrm{~cm}^{2} \mathrm{~s}^{-2}$ \\
$v \ldots \ldots \ldots \ldots$ & $3.86 \times 10^{5} \mathrm{~cm} \mathrm{~s}^{-1}$ \\
$v_{d} \ldots \ldots \ldots \ldots$ & $8.39 \times 10^{5} \mathrm{~cm} \mathrm{~s}^{-1}$ \\
$v_{g} \ldots \ldots \ldots \ldots$ & $1.23 \times 10^{6} \mathrm{~cm} \mathrm{~s}^{-1}$ \\
$\rho \ldots \ldots \ldots \ldots$ & $9.5 \times 10^{-17} \mathrm{~g} \mathrm{~cm}^{-3}$ \\
$T \ldots \ldots \ldots \ldots \ldots$ & $890 \mathrm{~K}$ \\
$T_{x} \ldots \ldots \ldots \ldots$ & $770 \mathrm{~K}$ \\
$\varepsilon \ldots \ldots \ldots \ldots$ & 2.17 \\
$d M^{2} / d \xi \ldots \ldots$ & 6.09 \\
$d H / d \xi \ldots \ldots$ & $1.74 \times 10^{11} \mathrm{~cm}^{2} \mathrm{~s}^{-2}$ \\
\hline
\end{tabular}




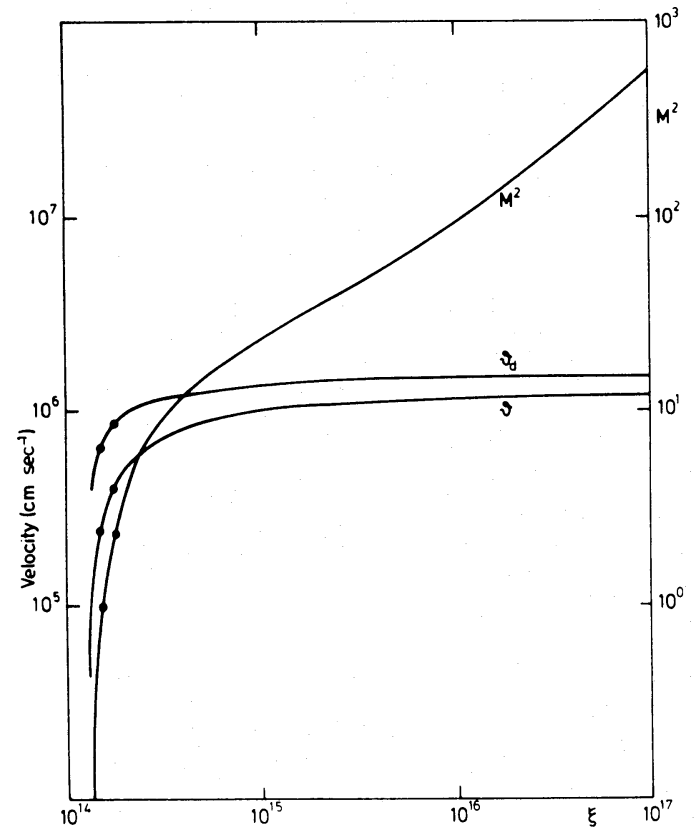

FIG. 2.-The velocity of the gas $(v)$, the drift velocity of the dust $\left(v_{d}\right)$, and the Mach number of the gas as a function of $\xi$. The dots indicate the sonic and critical points.

\section{b) Density Structure}

Going outward, the density initially drops much faster than expected on the basis of an $r$-squared law (see Fig. 3). When the gas has reached its terminal velocity, the density obviously follows such a law (cf. eq. [5]). The total mass column density, $N$, is about $2.4 \times 10^{-2}$ $\mathrm{g} \mathrm{cm}^{-2}$. If one had to calculate the mass loss rate from this column density, using the terminal velocity and the inner radius and assuming an $r$-squared dependence for the density, then the mass loss rate would be overestimated by about a factor of 3.5. The value of $N$ is, however, quite dependent on the exact value assumed for the inner radius, that is, the velocity at which the calculations are terminated (cf. eq. [5]). The dust-to-gas ratio, $\rho_{d} / \rho$, in the circumstellar shell varies because of the large drift velocity of the grains with respect to the gas (Fig. 3). This ratio is nowhere in the circumstellar shell equal to the value chosen for $\delta\left(=4 \times 10^{-3}\right)$. Again this is due to the difference in velocity between dust and gas (cf. eqs. [1] and [5]). This effect should be kept in mind in estimating mass loss rates from infrared continuum observations of circumstellar dust. The dust column density is $3 \times 10^{-5} \mathrm{~g} \mathrm{~cm}^{-2}$, and the ratio of dust column density to gas column density is $1.2 \times 10^{-3}$, which is much less than the value assumed for $\delta$. Since the drift velocity depends on the mass loss rate (cf. eq.

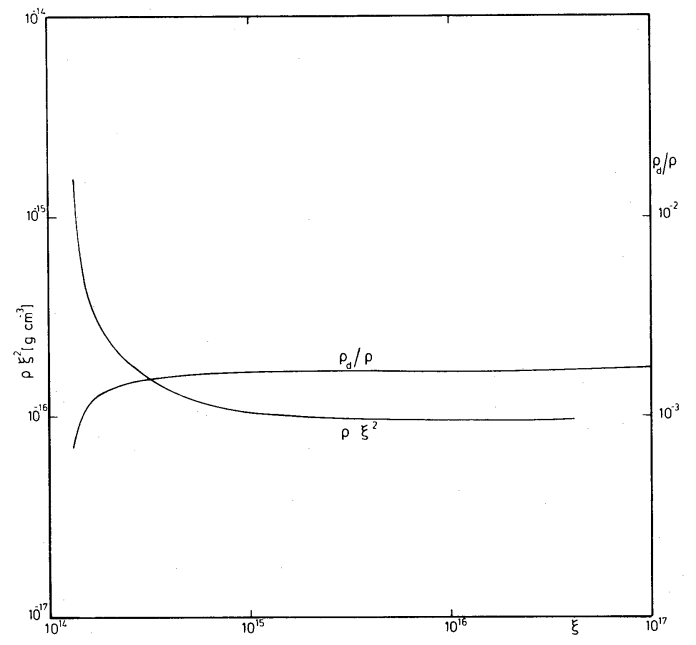

FIG. 3.-The gas density, $\rho$, and the dust-to-gas density ratio, $\rho_{d} / \rho$, as a function of $\xi$. The gas density is scaled by a factor $\xi^{2} / \xi_{0}^{2}$.

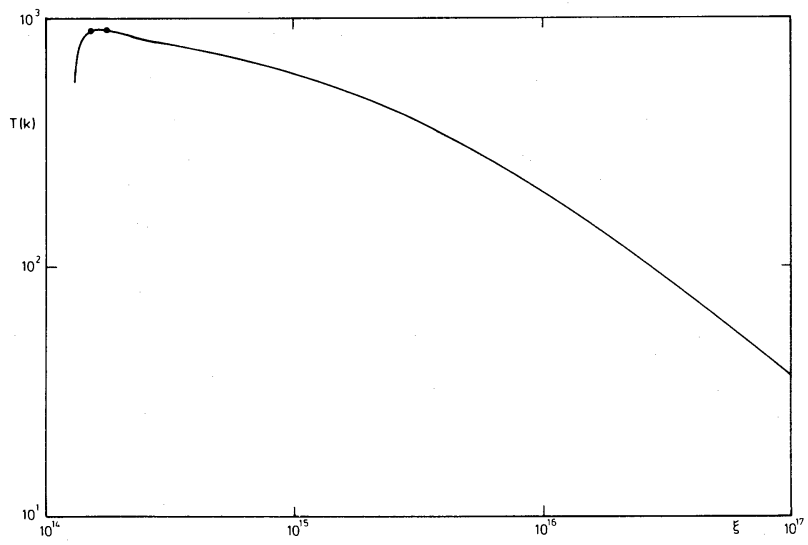

FIG. 4. - The kinetic temperature of the gas as a function of $\xi$. The dots indicate the sonic and critical points.

TABLE 3

CONDITIONS AT THE INNER BOUNDARY

\begin{tabular}{ll}
\hline \hline$\xi_{0} \ldots \ldots \ldots \ldots$ & $1.30 \times 10^{14}$ \\
$M_{0}^{2} \ldots \ldots \ldots \ldots$ & $5.0 \times 10^{-2}$ \\
$H_{0} \ldots \ldots \ldots \ldots$ & $6.0 \times 10^{10} \mathrm{~cm}^{2} \mathrm{~s}^{-2}$ \\
$v_{\ldots} \ldots \ldots \ldots \ldots$ & $4.4 \times 10^{4} \mathrm{~cm} \mathrm{~s}^{-1}$ \\
$v_{d} \ldots \ldots \ldots \ldots$ & $2.6 \times 10^{5} \mathrm{~cm} \mathrm{~s}^{-1}$ \\
$v_{g} \ldots \ldots \ldots \ldots$ & $3.0 \times 10^{5} \mathrm{~cm} \mathrm{~s}^{-1}$ \\
$\rho_{0} \ldots \ldots \ldots \ldots$ & $1.5 \times 10^{-15} \mathrm{~g} \mathrm{~cm}^{-3}$ \\
$T_{0} \ldots \ldots \ldots \ldots$ & $550 \mathrm{~K}$ \\
$T_{x} \ldots \ldots \ldots \ldots$ & $550 \mathrm{~K}$ \\
$\varepsilon \ldots \ldots \ldots \ldots$ & $3.12 \times 10^{1}$ \\
$d M^{2} / d \xi \ldots \ldots$ & 1.9 \\
$d H / d \xi \ldots \ldots \ldots$ & $5.7 \times 10^{11} \mathrm{~cm}^{2} \mathrm{~s}^{-2}$ \\
\hline
\end{tabular}


[12]), one may expect variations in the observed ratios of dust-to-gas column densities. Adopting a typical value for the strength of the $10 \mu \mathrm{m}$ feature in amorphous silicates $\left(3 \times 10^{3} \mathrm{~cm}^{2} \mathrm{~g}^{-1}\right.$; Day 1979), we infer a $10 \mu \mathrm{m}$ optical depth of about 0.1 .

\section{c) Temperature Structure}

After an initial rise, the temperature drops steadily (Fig. 4). In our calculations three competitive processes determine the temperature of the gas: the viscous heating by gas-grain collisions, the $\mathrm{H}_{2} \mathrm{O}$ rotational cooling, and the cooling due to expansion (cf. eq. [10]). The behavior of these terms as a function of $\xi$ is shown in Figure 5. The heating due to gas-grain collisions is more or less constant throughout the flow. The $\mathrm{H}_{2} \mathrm{O}$ rotational cooling initially increases rapidly, reaches a maximum, and then falls off again. Close to the inner boundary, the density is high. Because of the resulting low escape probability, only a small number of photons are able to escape. The excitation temperature is therefore equal to the kinetic temperature, and the cooling is small. Going outward the gas is accelerated rapidly, and the cooling increases. Far from the inner boundary the $\mathrm{H}_{2} \mathrm{O}$ rotational cooling decreases again, because fewer and fewer rotational levels of $\mathrm{H}_{2} \mathrm{O}$ can be populated. Furthermore, because of the drop in density, fewer and fewer $\mathrm{H}_{2} \mathrm{O}$ molecules are excited by collisions. For a constant acceleration, $\varepsilon$, the third term in equation (10) is just the cooling due to adiabatic expansion. In the absence of other heating and cooling terms and acceleration, this term would give rise to a $-4 / 3$ power dependence of temperature on the radius. As is obvious from Figure 5, this term decreases sharply in going outward from the inner radius because of the decrease in the velocity gradient. This decrease gives rise to the increase in temperature close to the stationary layer (Fig. 4). Far from the inner radius the temperature drops again,

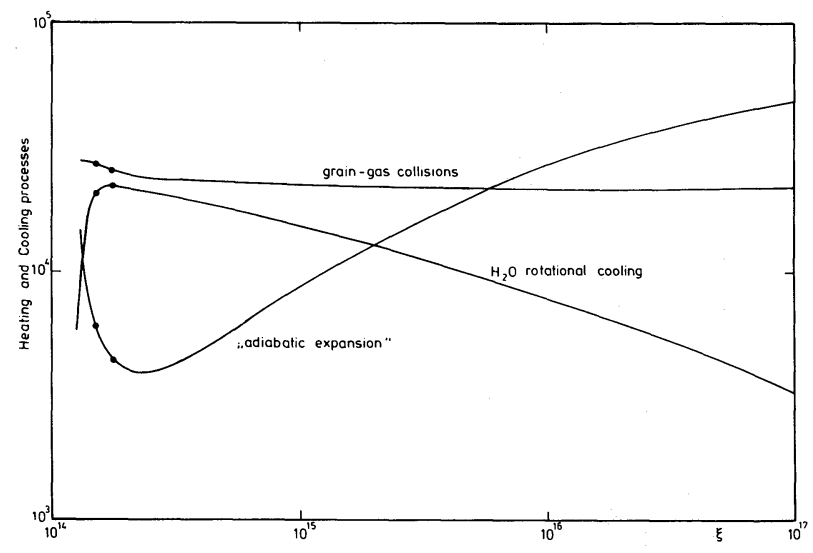

FIG. 5.-The behavior of the three different terms in the temperature gradient as a function of $\xi$. In order to display the changes better these terms are multiplied by $\xi^{2}$. basically because of the adiabatic expansion. However, nowhere in the flow does the temperature show a $-4 / 3$ power dependence on radius.

In our calculations of the heating function we have limited ourselves to heating due to gas-grain collisions and $\mathrm{H}_{2} \mathrm{O}$ rotational cooling. As was shown by Goldreich and Scoville (1976), the latter process will act as an energy source for the gas close to the star, because $\mathrm{H}_{2} \mathrm{O}$ molecules can be vibrationally excited by stellar photons. Subsequent radiative decay leaves the molecules in an excited rotational level of the ground vibrational state. Collisional de-excitation transfers the rotational energy then to translational energy of the gas. For simplicity we have ignored this process. Including it in the rate equation governing the level population will change the functional dependence of the excitation parameter, $x$, and the $\mathrm{H}_{2} \mathrm{O}$ rotational cooling, $Q_{2}$, on the velocity gradient (eq. [21]). However, the essential point of this calculation remains intact. The cooling will still depend on the velocity gradient, and qualitatively our analysis of the flow is still correct. Quantitatively there may be some differences.

We will now discuss two cooling processes suggested by Goldreich and Scoville (1976) which are not included in our calculation. Goldreich and Scoville (1976) identify chemical energy stored in the gas as an important cooling process of the gas. They consider, in particular, the reaction

$$
\mathrm{H}_{2}+\mathrm{OH} \rightarrow \mathrm{H}_{2} \mathrm{O}+\mathrm{H}(+0.7 \mathrm{eV}),
$$

which is exothermic by about $0.7 \mathrm{eV}$ in the forward direction. Part of the excess energy goes into vibrational excitation of the resulting $\mathrm{H}_{2} \mathrm{O}$ molecule. This fraction of the excess energy is lost for the gas since radiative decay will dominate over collisional de-excitation of the $\mathrm{H}_{2} \mathrm{O}$ molecule, and all the photons so produced will escape. However, in their definition of the internal energy of the gas, the chemically stored energy is not included. If it had been included, then the forward reaction would indeed decrease the internal energy of the gas. It should be noted, however, that the forward reaction will act as an energy source for the kinetic temperature of the gas since a considerable fraction of the chemical energy is carried away as translational energy of the product molecules. The backward reaction is, of course, a sink for the kinetic energy of the gas. The net result of the forward and backward reaction is that the gas is heated. From the results of Goldreich and Scoville (1976) we have estimated that this heating term is less important than the excitation of $\mathrm{H}_{2} \mathrm{O}$ molecules through absorption of stellar photons discussed above. Goldreich and Scoville (1976) also take into account collisional excitation of vibrational levels of $\mathrm{H}_{2}$ molecules followed by radiative decay. However, at the low 
kinetic temperatures $(T<1000 \mathrm{~K})$ we are interested in, this process can be neglected.

\section{d) The Importance of the Heating and Cooling Processes}

Our results described above show that for a qualitative description of the outflow the cooling of the gas should be included. It is of some interest to study the quantitative influence of the flow. We have therefore also calculated the structure of the outflow in the absence of $\mathrm{H}_{2} \mathrm{O}$ rotational cooling. In this calculation the gas is still heated by grain-gas collisions and cooled through adiabatic expansion.

In this calculation the critical point coincides with the sonic point (see § III $a$ ). Choosing the temperature at the sonic point, the radius is found from equation (22) using $M^{2}=1$. The acceleration at the sonic point follows then from equation 26. Finally, integrating inward yields the condition at the inner boundary. Through a trial and error procedure, the density at the inner boundary was made equal to the value found in the calculation including $\mathrm{H}_{2} \mathrm{O}$ rotational cooling (Table 3 ). The gas velocity is then automatically equal to the value in the previous calculation. The gas is now cooled only by adiabatic expansion. Its temperature at the inner boundary is therefore slightly higher than previously $\left(T_{0}=690 \mathrm{~K}\right)$. Choosing the temperature instead of the density as the boundary condition introduces only small differences in this calculation. It has no effect on the subsequent discussion.

Neglecting $\mathrm{H}_{2} \mathrm{O}$ rotational cooling has a profound influence on the structure of the flow. Obviously, the largest effect occurs in the temperature, which initially rises sharply to a maximum of about $3800 \mathrm{~K}$ at $\xi=3.3$ $\times 10^{14}$. Beyond that point it drops steadily and reaches a value of $50 \mathrm{~K}$ at $\xi=10^{14}$. The changes in the velocity structure are, however, also nonnegligible. The acceleration of the gas is much larger than in the previous calculation leading to a considerably larger terminal velocity $\left(18 \mathrm{~km} \mathrm{~s}^{-1}\right.$ versus $\left.12 \mathrm{~km} \mathrm{~s}^{-1}\right)$.

In order to understand this difference it is important to realize that grain-gas collisions drive the outflow in two ways. First, there is the direct momentum transport, described in $\S$ II $c$ (i). Second, the gas is heated (§ II $c[$ ii] $)$. This causes the gas to expand, that is, to convert its thermal energy into systematic kinetic energy. The larger terminal velocity is therefore not too surprising. The only way the gas can lose the viscous heat in this calculation is by adiabatic expansion. It should be noted that in this case the outflow is still mainly driven by process 1 . Process 2 contributes only about $20 \%$.

If we had neglected both the grain-gas viscous heating and the $\mathrm{H}_{2} \mathrm{O}$ rotational cooling, then no stationary solution for the gas outflow would exist. This can be shown in the following way (cf. Kwok 1975). In the absence of energy sources or sinks, the sonic condition implies that $\Gamma$ should be about unity at the sonic point (cf. eq. [22]). Using equations (11) and (12) and $M^{2}=1$, we find for the mass loss rate

$$
\dot{\mathfrak{T}}_{\star}=\frac{\bar{Q}_{\mathrm{rp}} L_{\star}}{c_{l} c}\left(\frac{\sigma_{g} \bar{Q}_{\mathrm{rp}} L_{\star} \delta}{4 \pi c_{l} G M_{\star} m_{d}}-1\right)^{-2} .
$$

Because the temperature is monotonically decreasing outward, this yields a lower limit for the mass loss rate for a given temperature at the inner boundary. Inserting numerical values, we find a minimum mass loss rate of $1.7 \times 10^{19} \mathrm{~g} \mathrm{~s}^{-1}$ for an inner boundary temperature of $550 \mathrm{~K}$. Our chosen value for the mass loss rate $\left(1.4 \times 10^{19}\right.$ $\mathrm{g} \mathrm{s}^{-1}$ ) is below this value, and consequently, no stationary solution to the flow exists. The physics behind this equation is that for certain inner boundary conditions the grain-gas coupling is too small for the gas to overcome the gravitational potential of the star. Including gas heating and cooling changes this considerably. Obviously, the condition at the critical point differs from the one used above because of the presence of heating and cooling terms (cf. eq. [22]). More importantly, the flow can adjust the temperature at the critical point. This lowers the lower limit to the mass loss rate. Clearly, both heating and cooling processes are of prime importance for a proper understanding of stellar winds.

\section{e) Some Further Remarks on the Flow}

In addition to the solution discussed above ( $\S \S$ IV $a-c$ ), we found for a limited range of critical radii another continuous flow solution. This solution starts supersonically at the inner boundary and decelerates smoothly to a subsonic flow at the outer boundary. This is somewhat surprising since, at first sight, one would expect that the radiation pressure on the grains will accelerate a flow. The second solution is the result of the viscous interaction between the dust and gas. Because of the large drift velocity at the inner boundary, the viscous heating of the gas is large. This gives rise to a negative pressure gradient and therefore decelerates the flow. Since the gas velocity decreases faster than the grain velocity (cf. eq. [12]), the ratio of radiation pressure to gravitational force, $\Gamma$, also decreases (cf. eq. [11]), and the flow will decelerate further. This kind of solution is, however, an artifact of our theory arising from neglecting grain formation at the inner boundary. In a theory which treats grain formation properly, this kind of solution does not exist.

Close to the inner boundary the ratio of the radiation pressure to the gravitational force, $\Gamma$, should go to zero because $n_{g}$ should go to zero. In the model calculation presented above, $\Gamma$ indeed does go to zero. This is, however, due to the fact that $\rho_{d} / \rho$ goes to zero (see Fig. 3 ) as a result of the large drift velocity of grains with 
respect to the gas. For increasing mass loss rates, the drift velocity will decrease (cf. eq. [12]). Consequently, for large enough mass loss rates $\left(>3 \times 10^{-6} M_{\odot} \mathrm{yr}^{-1}\right)$, $\Gamma$ will not go to zero at the inner boundary unless the kinetic temperature is very low. For a mass loss rate of $3 \times 10^{-5} M_{\odot} \mathrm{yr}^{-1}$, a kinetic temperature of about $10 \mathrm{~K}$ is needed for $\Gamma$ to become zero at the inner boundary. This is unacceptably low. Obviously, at these high mass loss rates, dust formation should be included if we want to attain an acceptable solution to the dynamical problem.

\section{f) Grain Formation}

The observational picture for the circumstellar envelope of late-type stars losing mass, described in the Introduction, has important consequences for our thoughts on dust formation. The small clusters which act as nucleation centers for the dust grains can probably not survive the high gas temperature $(T \approx 2000-4000$ $\mathrm{K})$ and the shocks in the extended pulsating photosphere. Dust particles can then only start to form in the stationary layer, where most of the activity has decayed already. At these large distances from the star the dust temperature will be less than the condensation temperature of the silicate material $\left(T_{\text {con }} \sim 1000 \mathrm{~K}\right)$. This is in agreement with infrared interferometric observations (Sutton et al. 1978). Because of the low temperature, more than one molecule might be able to condense out at the same nucleation center. The disorder in the solid produced by this and by the low condensation temperature will broaden the infrared vibration features of the solid particles (Day 1976; Day and Donn 1978). The impurity molecules which also have condensed out may give rise to the high absorptivity around $1 \mu \mathrm{m}$ postulated by Jones and Merrill (1976) and Bedijn (1977). Obviously, laboratory experiments on the condensation of a supercooled mixture resembling the one expected around Mira variables will be useful in testing this hypothesis.

The density in circumstellar envelopes might be high enough for other molecules to condense out on the silicate grains when they reach the condensation temperature of the particular molecule. The most abundant molecules after $\mathrm{H}_{2}$ are $\mathrm{H}_{2} \mathrm{O}$ and $\mathrm{CO}$. They have condensation temperatures of $90 \mathrm{~K}$ and $10 \mathrm{~K}$, respectively (Nakagawa 1980). Because the condensation temperature of $\mathrm{CO}$ is much lower than that of $\mathrm{H}_{2} \mathrm{O}$, the $\mathrm{H}_{2} \mathrm{O}$ will condense in the form of pure amorphous solid water $\mathrm{H}_{2} \mathrm{O}$ (as). The relatively high condensation temperature implies that $\mathrm{H}_{2} \mathrm{O}$ (as) will be partially annealed (Hagen, Tielens, and Greenberg 1981). Both the pure $\mathrm{H}_{2} \mathrm{O}$ (as) grain mantle as well as the high condensation temperature are in agreement with the shape of the $3250 \mathrm{~cm}^{-1}$ $(3.07 \mu \mathrm{m})$ absorption band observed in the spectrum of $\mathrm{OH} 231.8+4.2$ (Hagen, Tielens, and Greenberg 1982). For stars with a mass loss rate as low as in the model calculations discussed above, $\mathrm{H}_{2} \mathrm{O}$ grain mantles will not form. This is due to the low gas density at the condensation radius of $\mathrm{H}_{2} \mathrm{O}\left(\sim 10^{16} \mathrm{~cm}\right)$. Typically, a mass loss rate of about $10^{-5} M_{\odot} \mathrm{yr}^{-1}$ is needed for an appreciable $\mathrm{H}_{2} \mathrm{O}$ grain mantle to form. The $\mathrm{H}_{2} \mathrm{O}$ grain mantles will not survive long in the harsh surroundings of the normal interstellar medium. The time scale for the destruction of a pure $\mathrm{H}_{2} \mathrm{O}$ grain mantle due to photodesorption and due to sputtering in cloud-cloud collisions is estimated to be about $5 \times 10^{4}$ to $5 \times 10^{6} \mathrm{yr}$ (Barlow 1978; Draine and Salpeter 1979).

\section{g) The Outer Boundary}

In our analysis of the flow in the circumstellar shells of oxygen-rich Mira variables we have only taken into account the inner boundary conditions. Far from the inner boundary the stellar wind will merge with the interstellar medium. Since for large $\xi$ the density drops proportionally to $\xi$ squared, while the velocity becomes constant, there will be a point in the flow where the momentum transport by the flow, $\rho v^{2}$, becomes equal to the interstellar gas pressure, $p_{i}$. In the neighborhood of this point there will be a shock front (McCrea 1956; Parker 1960). Assuming an interstellar gas pressure of $2.6 \times 10^{-13} \mathrm{dyn} \mathrm{cm}^{-2}$ (Spitzer 1978), this condition yields a radius of the shock front in our model of $2.5 \times 10^{18}$ $\mathrm{cm}$. The time to reach this radius, $\sim 7 \times 10^{4} \mathrm{yr}$, is however, long compared with the mass loss phase. Consequently, the outflow of Mira variable will not have accommodated itself to the boundary condition at "infinity."

\section{h) Comparison with Previous Work}

In previous work the essential importance of the molecular cooling on the dynamics has not been considered, probably because previous investigators were studying the effects of dynamics on grain formation, chemistry, or infrared spectra. Because these problems are already very complicated by themselves, simplifying assumptions were introduced. They have either completely neglected the pressure gradient in the momentum equation (Olnon 1977), assumed an adiabatic relationship (Kwok 1975), assumed that the temperature is dominated by radiative processes (Menietti and Fix 1978), or even just assumed a velocity distribution (Goldreich and Scoville 1976). As our results show, the dynamics of the outflow are dominated by the cooling of the gas. For a proper assessment of the effects of dynamics on the other processes occurring in a circumstellar shell, the cooling should be included. In the future we hope to investigate the effects on the dynamics of varying the parameters involved in the calculations, e.g., the radius and temperature of the stationary layer and the properties of the dust. We expect that more and more detailed velocity and temperature in- 
formation will become available in the near future, and these calculations will be a useful guideline for observers. We also intend to study the effects of grain growth and radiative transfer on the dynamics.

\section{SUMMARY}

We can summarize our work as follows. A model for the stellar wind driven by radiation pressure on grains has been developed. This theory includes the essential aspect of the coupling between velocity and temperature structure of the gas. As in the familiar theory of the solar wind, there exists a critical point in the flow through which a stationary solution has to pass. For a typical Mira variable, this flow is calculated numerically, and its characteristics are discussed briefly. It is shown that, for a stellar wind driven by radiation pressure on dust grains, not only the momentum transfer but also the energy transfer by gas-dust collisions drives the gas outflow.

I would like to thank Dr. B. van Leer for the clarifying discussions on the flow equations and the properties of the critical point. I am grateful to Dr. F. Olnon for the many stimulating discussions on the outflows of Mira variables, for his meticulous checking of the algebra, and his Bokma. Dr. H. J. Habing originally made the suggestion of modeling the dynamics, radiative transfer, chemistry, and maser emission in the outflows of $\mathrm{M}$ giants in the last chapter of my thesis. I am indebted to him for this, for relaxing the criteria for this chapter in view of an impossible time limit, and for support during critical stages of my $\mathrm{Ph} . \mathrm{D}$. research.

\section{APPENDIX}

In this Appendix we will derive the equations describing the rotational cooling of $\mathrm{H}_{2} \mathrm{O}$ (eqs. [16] and [21]). We have represented the $\mathrm{H}_{2} \mathrm{O}$ molecule by a classical two-level rotor. For a good approximation of the excitation temperature and the cooling of circumstellar $\mathrm{H}_{2} \mathrm{O}$ by this model molecule, the value of the energy difference between the two levels $(h \nu)$ and the number of model molecules in the system $(n)$ should be chosen with care (Goldreich and Scoville 1976). The rms value of the angular velocity of the model molecule is (Landau and Lifshitz 1980)

$$
\omega=\left[k T_{x}\left(\frac{1}{I_{1}}+\frac{1}{I_{2}}+\frac{1}{I_{3}}\right)\right]^{1 / 2},
$$

where the $I_{i}$ are the molecular moments of inertia. The rotational frequency is thus

$$
\nu=\frac{\omega}{2 \pi} \equiv \nu_{0} T_{x}^{1 / 2}
$$

with $\nu_{0}$ a constant.

Our model molecule has $(2 J+1)$ rotational levels available, where the rotational quantum number $J$ is given by (Landau and Lifshitz 1980)

$$
J=\frac{1}{h}\left[k T_{x}\left(I_{1}+I_{2}+I_{3}\right)\right]^{1 / 2}
$$

A circumstellar $\mathrm{H}_{2} \mathrm{O}$ molecule has $Z_{\text {rot }}$ rotational levels available at an excitation temperature, $T_{x}$, where the rotational partition function, $Z_{\text {rot }}$, is given by (Landau and Lifshitz 1980)

$$
Z_{\text {rot }}=\frac{\left(2 k T_{x}\right)^{3 / 2}\left(\pi I_{1} I_{2} I_{3}\right)^{1 / 2}}{2 h^{3}}
$$

The appropriate value of $n$ is thus

$$
n=\frac{2(2 J+1)}{Z_{\mathrm{rot}}} n_{\mathrm{H}_{2} \mathrm{O}} \approx \frac{4 J}{Z_{\mathrm{rot}}} n_{\mathrm{H}_{2} \mathrm{O}} .
$$

With equations (A1) and (A2), equation (15) can now be rewritten to equation (16), and equations (17)-(20) to equation (21). 
Abbott, D. C. 1977, Ph.D. thesis, University of Colorado. Bärlow, M. J. 1978, M.N.R.A.S., 183, 397.

Bedijn, P. 1977, Ph.D. thesis, University of Leiden

Bernat, A. P., Hall, D. N. B., Hinkle, K. H., and Ridgway, S. T. 1979, Ap. J. (Letters), 233, L135.

Betz, A. L., and McLaren, R. A. 1980, in IAU Symposium 87, Interstellar Molecules, ed. B. H. Andrew (Dordrecht: Reidel), p. 503.

Bondi, H. 1952, M.N.R.A.S., 112, 195.

Cassinelli, J. P. 1979, Ann. Rev. Astr. Ap., 17, 275.

Castor, J. I. 1970, M.N.R.A.S., 149, 111.

Castor, J. I., Abbott, D. C., and Klein, R. I. 1975, Ap. J., 195, 157

Clegg, R. S., van IJzendoorn, L. J., and Allamandola, L. J. 1982, preprint.

Couturier, P., Mangeney, A., and Souffrin, P. 1979, Astr. Ap., 74, 9.

Day, K. L. 1976, Ap. J. (Letters), 203, L99. 1979, Ap. J., 234, 158.

$\overline{\text { Day, K}}$. L., and Donn, B. 1978, Ap. J. (Letters), 222, L45.

Deguchi, S. 1980, Ap. J., 236, 567.

Deutsch, A. J. 1956, Ap. J., 123, 210

Dickinson, D. F., and Chaisson, E. J. 1973, Ap. J. (Letters), 181, L135.

Dickinson, D. F., Kollberg, E., and Yngnesson, S. 1975, Ap. J., 199, 131.

Draine, B. T., and Salpeter, E. E. 1977, J. Chem. Phys., 67, 2330. 1979, Ap. J., 231, 438

Elitzur, M., Goldreich, P., and Scoville, N. 1976, Ap. J., 205, 384.

Forrest, W. J., et al. 1978, Ap. J., 219, 114.

Gehrz, R. D., and Woolf, N. J. 1971, Ap. J., 165, 285.

Gilman, R. C. 1972, Ap. J., 178, 423.

Goldreich, P., and Scoville, N. 1976, Ap. J., 205, 144

Hagen, W., Tielens, A. G. G. M., and Greenberg, J. M. 1981, Chem. Phys., 56, 367. 1982, Astr. Ap., 117, 132.

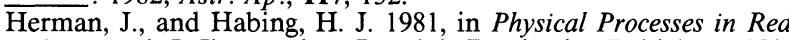
Giants, ed. I. Iben and A. Renzini (Dordrecht: Reidel), p. 383

Hill, S. J., and Willson, L. A. 1979, Ap. J., 229, 1029.

Hinkle, K. H. 1978, Ap. J., 220, 210.

Hinkle, K. H., and Barnes T. G. 1979, Ap. J., 227, 923.

Hinkle, K. H., Hall, D. N., and Ridgway, S. T. 1982, preprint

Holzer, T. E., and Axford, W. I. 1970, Ann. Rev. Astr. Ap., 8, 31

Jewell, P. R., Webber, J. C., and Snyder, L. E. 1980 Ap. J. (Letters), 242, L2.9.

Jones, T. W., and Merrill, K. M. 1976, Ap. J., 209, 509.

Kwok, S. 1975, Ap. J., 198, 583.

Landau, L. D., and Lifshitz, E. M. 1980, Course of Theoretical Physics, Vol. 5, Statistical Physics, Part 1 (Oxford: Pergamon).

\section{RFERENCES}

Lo, K. Y., and Bechis, K. P. 1977, Ap. J. (Letters), 218, L27.

McCrea, H. C. 1956, Ap. J., 124, 437.

Menietti, J. D., and Fix, J. D. 1978, Ap. J., 224, 961

Merrill, K. M. 1977, in IAU Colloquium 42, The Interaction of Variable Stars with Their Environment, ed. R. Kippenhahn, J. Rahe, and W. Strohmeier (Veröf. Remeis-Sternw. Bamberg, Vol. 11, No. 121), p. 446.

Moran, J. M., Ball, J. A., Predmore, C. R., Lane, A. P., Huguenin, G. R., Reid, M. J., and Hansen, S. S. 1979, Ap. J. (Letters), 231, L67.

Morris, M., Redman, R., Reid, M. J., and Dickinson, D. F. 1979, Ap. J., 229, 257.

Nakagawa, N., 1980, in IAU Symposium 87, Interstellar Molecules, ed. B. H. Andrew (Dordrecht: Reidel), p. 365.

Olnon, F. M. 1977, Ph.D. thesis, University of Leiden.

Parker, E. N. 1960, Ap. J., 132, 175.

Penman, J. M. 1976, M.N.R.A.S., 175, 149

Pollack, J. B., Toon, O. B., and Khare, B. N. 1973, Icarus, 19, 372.

Salpeter, E. E. $1974 a, A p . J ., 193,579$. $1974 b, A p . J ., 193,585$

Scalo, J. M., and Slavski, D. B. 1980, Ap. J. (Letters), 239, L73.

Sobolev, V. V. 1960, Moving Envelopes of Stars (Cambridge, Mass.: Harvard University Press).

Spencer, J. H., Johnston, K. J., Moran, J. M., Reid, M. J., and Walker, R. C. $1979, A p . J ., 230,449$.

Spitzer, L., Jr. 1978, Physical Processes in the Interstellar Medium (New York: Wiley).

Sutton, E. C., Storey, J. W. V., Townes, C. H., and Spears, D. L. 1978, Ap. J. (Letters), 224, L123.

Toombs, R. I., Becklin, E. E., Frogel, J. A., Low, S. K., Porter, F. C., and Westphal, J. A. 1972, Ap. J. (Letters), 173, L71.

Townes, C. H., and Schawlow, A. 1955, Microwave Spectroscopy, (New York: McGraw-Hill).

Tsuij, T. 1973, Astr. Ap., 23, 411.

Wallerstein, G. 1975, Ap. J. Suppl., 29, 375.

Watson, W. D., Elitzur, M., and Bieniek, R. J. 1980, Ap. J., 290, 547.

Weyman, R. 1962, Ap. J., 136, 844.

Willson, L. A., and Hill, S. J. 1979, Ap. J., 228, 854

Winnberg, A. 1977, in IAU Colloquium 42, The Interaction of Variable Stars with Their Environment, ed. R. Kippenhahn, J. Rahe, and W. Strohmeier (Veröff Reimeis-Sternw. Bamberg, Vol. 11, No. 121), p. 495

Wood, P. R. 1979, Ap. J., 227, 220.

Zuckerman, B., Gilra, D. P., Turner, B. E., Morris, M., and Palmer, P. 1976, Ap. J. (Letters), 205, L15.

Zuckerman, B., Palmer, P., Gilra, D. P., Turner, B. E., and Morris, M. 1978, Ap. J. (Letters), 220, L53.

A. G. G. M. TIElens: NASA Ames Research Center, MS 245-6, Moffett Field, CA 94035 